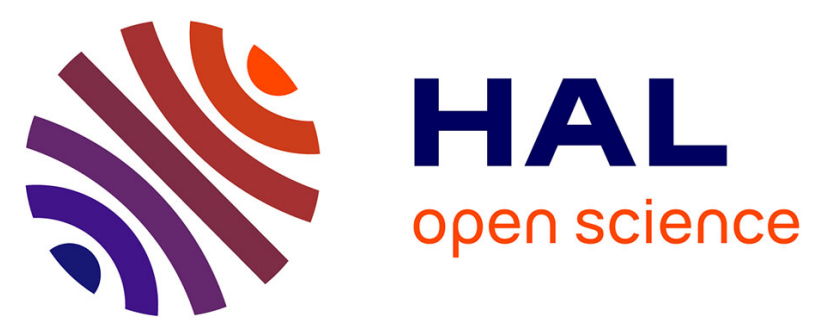

\title{
Multi-scale domain decomposition method for large scale structural analysis with a zooming technique: Application to plate assembly
}

\author{
Ahmad Mobasher Amini, David Dureisseix, Patrice Cartraud
}

\section{To cite this version:}

Ahmad Mobasher Amini, David Dureisseix, Patrice Cartraud. Multi-scale domain decomposition method for large scale structural analysis with a zooming technique: Application to plate assembly. International Journal for Numerical Methods in Engineering, 2009, 79 (4), pp.417-443. 10.1002/nme.2565 . hal-00401727

\section{HAL Id: hal-00401727 \\ https://hal.science/hal-00401727}

Submitted on 17 Jan 2017

HAL is a multi-disciplinary open access archive for the deposit and dissemination of scientific research documents, whether they are published or not. The documents may come from teaching and research institutions in France or abroad, or from public or private research centers.
L'archive ouverte pluridisciplinaire HAL, est destinée au dépôt et à la diffusion de documents scientifiques de niveau recherche, publiés ou non, émanant des établissements d'enseignement et de recherche français ou étrangers, des laboratoires publics ou privés.

\section{(ㅇ)(1) $\$$}

Distributed under a Creative Commons Attribution - NonCommercial - NoDerivatives $\mid 4.0$ 


\title{
Multi-scale domain decomposition method for large scale structural analysis with a zooming technique: Application to plate assembly
}

\author{
A. Mobasher Amini ${ }^{1}$, D. Dureisseix ${ }^{2}$, and P. Cartraud ${ }^{1}$ \\ ${ }^{1}$ GeM, École Centrale de Nantes / CNRS UMR 6183, 1 rue de la Noë, BP 92101, F-44321 \\ Nantes CEDEX 3, FRANCE \\ ${ }^{2}$ LMGC, Université Montpellier 2 / CNRS UMR 5508, CC048, Place E. Bataillon, F-34095 \\ Montpellier CEDEX 5, FRANCE
}

\begin{abstract}
This article is concerned with a multi-scale domain decomposition method, based on the FETI-DP solver, for large-scale structural elastic analysis and suited to problems that exhibit structural heterogeneities, such as plate assemblies in the presence of structural details. In this approach once a partition of the global fine mesh into subdomains has been performed (all subdomains possess a fine mesh) and to optimize the computational time, the fine mesh is preserved only in the zones of interest (with local phenomena due to discontinuity, hole, etc.) while the remaining subdomains are replaced by numerical homogenized coarse elements. Indeed, the multi-scale aspect is introduced by the description of subdomains with either a fine or a coarse scale mesh. As a result, an extension of the FETI-DP domain decomposition method is proposed in this article (called herein FETI-DP micro-macro) that allows the simultaneous usage of different discretizations: fine (microscopic) mesh for subdomains in zones of interest and coarse (macroscopic or homogenized) mesh for the complementary part of the structure. Using this strategy raises the problem of the determination of the stiffness of coarse subdomains, and of the incompatible finite element connection between fine and coarse subdomains. Two approaches (collocation and Mortar) are presented and compared. The article ends with patch tests, and some numerical examples in $2 \mathrm{D}$ and $3 \mathrm{D}$. The obtained numerical results exemplify the efficiency and capability of the FETI-DP micro-macro approach and reveal that the Mortar approach is more accurate, at constant cost, than the collocation approach.

This is the preprint of the following article: Ahmad Mobasher Amini, David Dureisseix, Patrice Cartraud, Multi-scale domain decomposition method for large scale structural analysis with a zooming technique: Application to plate assembly, International Journal for Numerical Methods in Engineering 79(4):417-443, Wiley, 2009, DOI: 10.1002/nme.2565, which has been published in final form at http://doi.org/10.1002/nme.2565
\end{abstract}

Keywords: Domain Decomposition Method, FETI-DP, Multi-scale, homogenization, structural heterogeneities, Mortar method

\section{INTRODUCTION}

The structural design of complex structures now often relies on finite element simulations. In the case of a large structure with small-size structural details, the finite element analysis is a difficult problem. To obtain a solution for the whole structure as well as a good accuracy near the structural details, a model with a fine mesh is required; this approach leads to a huge global finite element model with a large number of unknowns that is difficult to solve.

In the last years, many researches have been conducted to develop efficient numerical methods those are capable of solving large-scale problems. The direct sparse (out-of-core) solvers are robust, efficient and are already employed in several commercial finite element codes. These solvers need large memory resources and have a limited parallel scalability. The classical iterative solvers (Jacobi, Gauss Seidel, Conjugate Gradient, 
etc. [1]) are excellent from the memory usage point of view and can be easily parallelized [2]. But, their efficiency depends on the type of problem considered, and are not the most suited methods for mechanical engineering problems that are often ill-conditioned. Multigrid methods, see [3, 4], take advantage of using different description levels within an iterative approach (sometimes, coarse levels can be built automatically, as in smoothed aggregation methods [5]). Usually the coarsening ratio between two successive levels is somehow small; this leads to the use of many different levels to bridge the gap between microscopic and macroscopic scales.

An alternative choice is the domain decomposition methods (DDM), which combine advantages of both direct and iterative solvers. The main idea is based on the splitting of a large-scale domain into several subdomains with either overlapping or non-overlapping interfaces. For domain decomposition methods with non-overlapping interfaces, three approaches have been intensively studied. Based on parameters chosen on the interfaces, to enforce the continuity between the neighboring subdomains, these approaches are named as the primal, dual and mixed domain decomposition method, respectively [6]. The primal method chooses displacements as interface unknowns (Balancing Domain Decomposition method, or BDD, [7]). The dual method chooses interface forces as unknowns (classically dealt with as Lagrange multipliers in the FETI, [8] family of algorithms). In the mixed method where the interfaces play a major role, both displacements and forces are unknowns (LATIN method, [9], and in a less extend [10, 11]).

Dual-primal FETI (FETI-DP) [12,13] is the latest generation of the FETI methods that preserves the numerical and parallel scalability of the original FETI and FETI-2 [14] methods for second and fourth-order problems. This method also uses Lagrange multipliers to satisfy the continuity constraints on the interfaces. Indeed, instead of introducing the coefficients for rigid body modes in the original FETI and the second set of Lagrange multipliers in FETI-2, it chooses some 'corner node' degrees of freedom as basic unknowns so that each subdomain is non-singular [15]. The coarse problem of FETI-DP, which is essential for reaching scalability properties, is also sparser than the coarse problem of the method FETI or FETI-2. These features make FETI-DP method much more robust and applicable for implementation than its previous versions. The numerical experiments show that it also delivers better computational performance for most cases [12, 13].

In the domain decomposition method based iterative solvers, a direct sparse solver is used as the local solver in each subdomain. During this step, local forward and backward substitutions consume somehow a large percentage of the total CPU time. The total number of local resolutions depends almost linearly on the number of subdomains when the Dirichlet optimal preconditioner is used.

Therefore, the computational cost can be optimized by reducing the time spent in the local resolution step. In this article, we propose a method using the macro (homogenized) description of the subdomains which are not directly in the zones of interest. This algorithm requires a preliminary local homogenization step on the fine mesh of the subdomains, and removes unnecessary local factorizations on every subdomain in the coarse zones. Additionally, this algorithm reduces the size of the interface condensed problem and removes the unnecessary forward and backward substitution steps in the coarse zone, to save computational cost. Nevertheless, an overhead due to the numerical homogenization step is introduced by this strategy. This overhead is problem dependent, but in general, for large scale problems, it is acceptable and does not prevent savings of overall CPU time costs.

In the framework of domain decomposition method with incompatible meshes, several approaches have already been proposed in the literature, see e.g. [16], [17], [18] and references herein. However, in these works, the point was the coupling of different discretizations across subdomain interface. In this article, starting with a domain decomposition with matching interface, non-matching interfaces arise from the homogenization of subdomains. This method is called FETI-DP micro-macro throughout this article, and is developed in an elasticity framework.

For this purpose, this article is organized as follows. In Section 2, the general framework of the FETIDP method is briefly recalled. In Section 3, the main steps of the FETI-DP micro-macro approach are presented. Section 4 introduces the essentials of two connection methods (collocation and Mortar) and Section 5 concerns the determination of the macro (homogenized) stiffness of the macro zone. Section 6 presents the new interface problem of the FETI-DP micro-macro approach with two discretization scales for the subdomains. In Section 7, the method is validated with patch tests, and several examples and results are discussed. Finally, Section 8 concludes the article and suggests several future works. 


\section{DOMAIN DECOMPOSITION APPROACH}

\subsection{Selection of a domain decomposition method}

Domain decomposition methods are both efficient and flexible tools for structural analysis [19, 2]. When the size of the model increases, the iterative resolution methods, e.g. conjugate gradient (CG) method, can be used to solve the problem. They often allow a parallel treatment of the resolution phase. This article is not concerned with the parallelization of the resolution, but focus on the modularity of the methods, especially for coupling different subdomains that may have been modeled with different discretization levels.

The different versions of the FETI method (FETI-1 [8], FETI-2 [14], FETI-DP [12, 13]) belongs to the family of the non-overlapping domain decomposition methods with Lagrange multipliers. These methods have been developed as iterative solvers for large-scale systems of equations in structural analysis, obtained by using the finite element method. Among all the domain decomposition methods that were presented by several authors, the FETI-DP method is chosen here as a basis of development for the following reasons. Usually with a multi-scale domain decomposition method, the reference problem is split into subdomains and the coarse space problem is numerically built from this fine scale description. The coarse problem can be discrete by nature, and is not always related to any finite element model.

Within a micro-macro approach, as detailed in 3, the coarse nodes of the FETI-DP method may be used to define a coarse subdomain, which constitutes a structural homogenization of the detailed subdomain. In that way, the coarse compatibility of displacements are ensured automatically between neighboring coarse subdomains.

\subsection{Basic FETI-DP method}

In this Section, the FETI-DP method, as presented in $[12,13]$ is briefly reviewed to keep this article selfcontained, and to define the notations that will be used hereafter.

Let us consider the domain $\Omega$, partitioned into a set of $N_{s}$ non-overlapping subdomains $\Omega^{s}$. The subdomain-related nodes are classified into three groups: corner nodes $(c)$, non-corner interface nodes $(b)$ and the remaining internal nodes $(i)$, as well as their related degrees of freedom, see Figure 1.

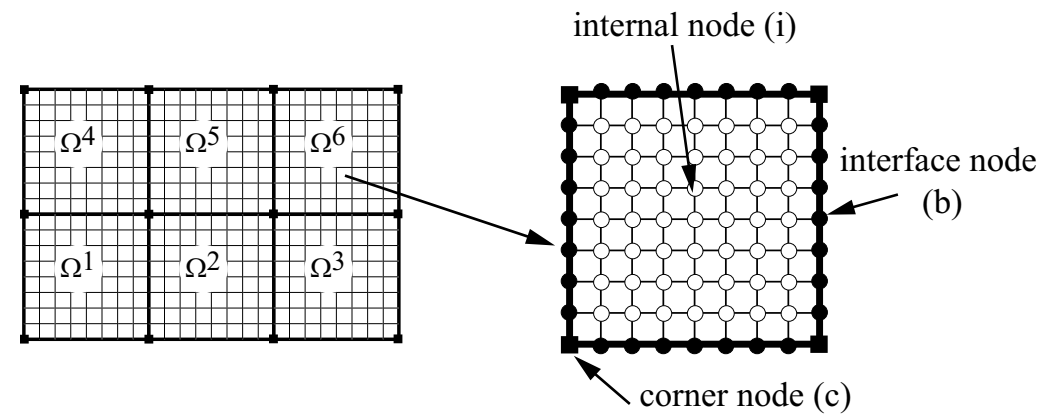

Figure 1: Classification of the subdomain nodes

The assembled stiffness matrix $K^{s}$, the solution vector $u^{s}$ and the loading vector $f^{s}$ on each subdomain can be split as follows:

$$
K^{s}=\left[\begin{array}{ccc}
K_{i i}^{s} & K_{i b}^{s} & K_{i c}^{s} \\
K_{b i}^{s} & K_{b b}^{s} & K_{b c}^{s} \\
K_{c i}^{s} & K_{c b}^{s} & K_{c c}^{s}
\end{array}\right], \quad u^{s}=\left[\begin{array}{c}
u_{i}^{s} \\
u_{b}^{s} \\
u_{c}^{s}
\end{array}\right], \quad f^{s}=\left[\begin{array}{c}
f_{i}^{s} \\
f_{b}^{s} \\
f_{c}^{s}
\end{array}\right]
$$

Denoting with $u_{c}$ the global vector of corner degrees of freedom, the global vector of degrees of freedom, 
$u$, and the subdomain-related vector of non-corner degrees of freedom, $u_{r}^{s}$, are defined as:

$$
u=\left[\begin{array}{c}
u_{r} \\
u_{c}
\end{array}\right]=\left[\begin{array}{c}
u_{r}^{1} \\
\cdot \\
\cdot \\
\cdot \\
u_{r}^{N_{s}} \\
u_{c}
\end{array}\right], \quad u_{r}^{s}=\left[\begin{array}{c}
u_{i}^{s} \\
u_{b}^{s}
\end{array}\right]
$$

Using these notations, one can also split the subdomain stiffness matrix into:

$$
K^{s}=\left[\begin{array}{ll}
K_{r r}^{s} & K_{r c}^{s} \\
K_{r c}^{s^{T}} & K_{c c}^{s}
\end{array}\right]
$$

Moreover, the continuity of the global solution is enforced at corner nodes. Two mapping matrices, $B_{r}^{s}$ and $L_{c}^{s}$, are introduced to locate the corresponding degrees of freedom. Here, $B_{r}^{s}$ are signed boolean matrices locating the non-corner degrees of freedom of the subdomain $\Omega^{s}$ that belongs to the global interface, and $L_{c}^{s}$ are localization matrices that give the corner degrees of freedom of each subdomain, from the global vector of corner degrees of freedom: $u_{c}^{s}=L_{c}^{s} u_{c}$.

Expressing the equilibrium equation for each subdomain in a global variational form leads to:

$$
\begin{aligned}
& K_{r r}^{s} u_{r}^{s}+K_{r c}^{s} L_{c}^{s} u_{c}+B_{r}^{s^{T}} \lambda=f_{r}^{s} \quad \text { for } s=1, \ldots, N_{s} \\
& \sum_{s=1}^{N_{s}} L_{c}^{s^{T}} K_{r c}^{s^{T}} u_{r}^{s}+\sum_{s=1}^{N_{s}} L_{c}^{s^{T}} K_{c c}^{s} L_{c}^{s} u_{c}=\sum_{s=1}^{N_{s}} L_{c}^{s^{T}} f_{c}^{s}=f_{c}
\end{aligned}
$$

with Lagrange multipliers $\lambda$ on the global interface, that traduce forces used to enforce the interface continuity condition:

$$
\sum_{s=1}^{N_{s}} B_{r}^{s} u_{r}^{s}=0
$$

To guaranty the non-singularity of the $K_{r r}^{s}$, it is important that each subdomain contains at least three non colinear corner nodes, [15].

With the above equations and after some algebraic transformations, the following dual-primal problem is obtained (using Lagrange multipliers $\lambda$, and primal corner displacement $u_{c}$, as unknowns):

$$
\left[\begin{array}{cc}
F_{I_{r r}} & F_{I_{r c}} \\
F_{I_{r c}}^{T} & -K_{c c}^{\star}
\end{array}\right]\left[\begin{array}{c}
\lambda \\
u_{c}
\end{array}\right]=\left[\begin{array}{c}
d_{r} \\
-f_{c}^{\star}
\end{array}\right]
$$

where

$$
\begin{array}{lr}
F_{I_{r r}}=\sum_{s=1}^{N_{s}} B_{r}^{s} K_{r r}^{s^{-1}} B_{r}^{s^{T}} & F_{I_{r c}}=\sum_{s=1}^{N_{s}} B_{r}^{s} K_{r r}^{s^{-1}} K_{r c}^{s} L_{c}^{s} \\
d_{r}=\sum_{s=1}^{N_{s}} B_{r}^{s} K_{r r}^{s^{-1}} f_{r}^{s} & f_{c}^{\star}=f_{c}-\sum_{s=1}^{N_{s}} L_{c}^{s^{T}} K_{r c}^{s^{T}} K_{r r}^{s^{-1}} f_{r}^{s} \\
K_{c c}=\sum_{s=1}^{N_{s}} L_{c}^{s^{T}} K_{c c}^{s} L_{c}^{s} & \\
K_{c c}^{\star}=K_{c c}-\sum_{s=1}^{N_{s}}\left(K_{r c}^{s} L_{c}^{s}\right)^{T} K_{r r}^{s^{-1}}\left(K_{r c}^{s} L_{c}^{s}\right) &
\end{array}
$$

By condensing $u_{c}$ on $\lambda$ in the equation (6), the following symmetric positive definite dual interface problem is obtained:

$$
F_{I} \lambda=D_{r}
$$


with:

$$
\begin{aligned}
& F_{I}=F_{I_{r r}}+F_{I_{r c}} K_{c c}^{\star^{-1}} F_{I_{r c}}^{T} \\
& D_{r}=d_{r}-F_{I_{r c}} K_{c c}^{\star^{-1}} f_{c}^{\star}
\end{aligned}
$$

The interface problem (8) is usually not solved by a direct method. Since in this case, the matrix $F_{I}$ and the vector $D_{r}$ should be assembled and for large-scale problems, assembling the interface problem can require unaffordable storage and computational resources. Moreover, assembling and solving the interface problem in a direct way would be a bottleneck for the parallel performance of the method.

The FETI-DP method can be viewed as the transformation of the global problem $K u=f$, into the interface problem (8), and a resolution with a iterative, e.g. conjugate gradient (CG), algorithm. This method does not need to explicitly assemble the interface operators (9). The main cost lies in the factorization of the subdomain matrices $K_{r r}^{s}$, and the use of matrix-vector products. Each matrix-vector product can be efficiently performed using subdomain by subdomain sparse matrix-vector product and local forward and backward substitutions.

From an implementation point of view, solving the interface problem (8) leads to the following steps:

- step 1:

$$
\delta^{k}=F_{I_{r r}} \lambda^{k}=\sum_{s=1}^{N_{s}} B_{r}^{s} K_{r r}^{s^{-1}} B_{r}^{s^{T}} \lambda^{k}
$$

- step 2:

$$
\delta^{k} \leftarrow \delta^{k}+F_{I_{r c}} K_{c c}^{\star^{-1}} F_{I_{r c}}^{T} \lambda^{k}
$$

Step 1 is the same as for the FETI method (local computation on each subdomain and assembling). Step 2 can be presented in three sub-steps as follows:

- Step 2.1:

$$
y^{k}=F_{I_{r}}^{T} \lambda^{k}=\sum_{s=1}^{N_{s}} L_{c}^{s^{T}} K_{r c}^{s^{T}} K_{r r}^{s^{-1}} B_{r}^{s^{T}} \lambda^{k}
$$

- Step 2.2: Solve $K_{c c}^{\star} x^{k}=y^{k}$ to get $x^{k}$

- Step 2.3:

$$
z^{k}=F_{I_{r c}} x^{k}=\sum_{s=1}^{N_{s}} B_{r}^{s} K_{r r}^{s^{-1}} K_{r c}^{s} L_{c}^{s} x^{k}
$$

Steps 2.1 and 2.3 correspond to local computations in each subdomain. The product $K_{r r}^{s^{-1}} B_{r}^{s^{T}} \lambda^{k}$ is already performed in Step 1 and the product $K_{r r}^{s^{-1}} K_{r c}^{s}$ is pre-computed in a preliminary step, when constructing $K_{c c}^{\star}$. Step 2.2 is the coarse space resolution of the mutilevel domain decomposition. This problem is solved at each iteration, for which it propagates globally the information among all the subdomains. Such a global problem is mandatory to reach scalability [20]. Note that for 3D problems arising for second-order partial differential equations, to recover scalability, an enrichment of the coarse space of FETI-DP method is required [12]. The matrix $K_{c c}^{\star}$ is sparse; its pattern is that of a stiffness matrix obtained by considering only the coarse super-elements defined on coarse nodes on each subdomain.

\section{FETI-DP MICRO-MACRO DESCRIPTION}

Depending on the problem and on the design process, if a global fine mesh is available, it can be splitted artificially into subdomains. In such a case, if a classical domain decomposition method is applied, each subdomain possesses its own fine mesh, which are compatible on the interface. When the local effects (stress concentration, discontinuity, etc.) are limited to few zones, this approach can be improved, since a quite large percentage of the CPU time is consumed by the local resolution on subdomains, i.e. computation of $K_{r r}^{s^{-1}} q^{k}$ where $q^{k}$ is a vector in equations (10), (12) and (13). The detailed analysis of the CPU time profiles depends 
on the number of subdomains, the size of the coarse problem, and the selection of the preconditioner. Some CPU time reports can be found in [14] and [21]. According to these references, $55 \%$ to $65 \%$ of the total $\mathrm{CPU}$ time is consumed by local resolutions. The reduction of these computations will therefore significantly improve the computational performance.

A multi-scale domain decomposition approach with different levels of discretization in subdomains is proposed herein: one considers the fine mesh only into the subdomains with local effects that the designer decided to check. The subdomains in zones of interest are therefore meshed finely (microscopic level) and subdomains located in the remaining areas of the structure are described with a coarse mesh only (macroscopic or homogenized level), see Figure 2.
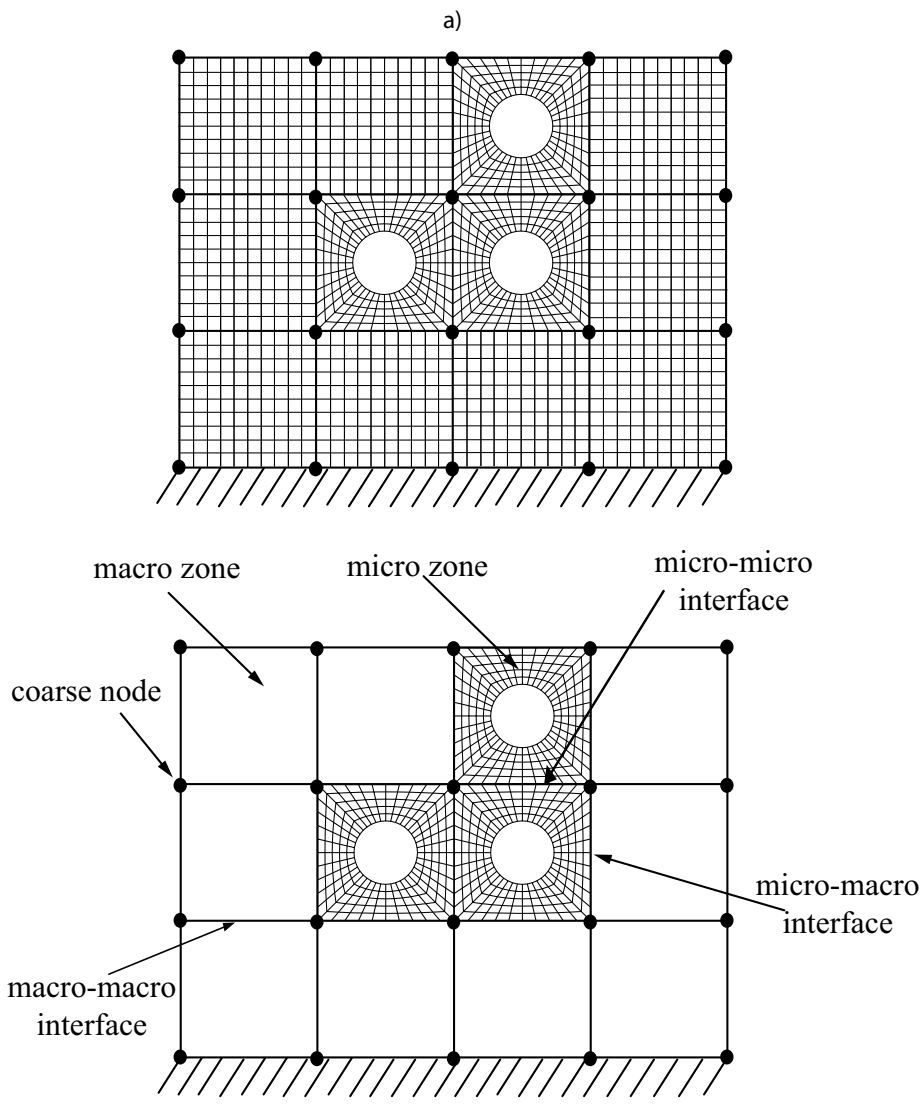

b)

Figure 2: Different levels of description: a) fine meshes in all the subdomains b) mixing fine and coarse descriptions

In order to develop a multi-scale domain decomposition method, one has to tackle three issues: (i) the choice of the micro and macro zones, (ii) the connection of fine and coarse meshes (incompatible mesh connection) on the subdomain interfaces and (iii) the determination of the macro stiffness.

It must be mentioned that in this work, the choice of the fine and coarse mesh is not automated, but rely on the expertise of the user. An important feature, not developed herein, is the possibility of switching the zoomed areas of interest on-the-fly during the analysis. Since the process is iterative, it won't need to be restarted from scratch, but would be designed in an adaptive framework. This aspect of the approach is currently under development. The issues (ii) and (iii) will be addressed in next Sections.

\section{FINE AND COARSE MESH CONNECTION METHOD}

The classical FETI-DP method is based on the perfect continuity of the displacement field on the interface, (5). When different discretizations in the subdomains are considered, the problem of incompatible connection 
of meshes appears at the micro-macro interface and the interface connections have to be reformulated, see Figure 2.

Let us consider two neighbouring subdomains $\Omega^{f}$ and $\Omega^{c}$ (fine and coarse, respectively) that are connected on a local interface $\Gamma$, see Figure 3. Along this interface, the coarse subdomain shares only two corner nodes, which are common to the finely meshed subdomain.

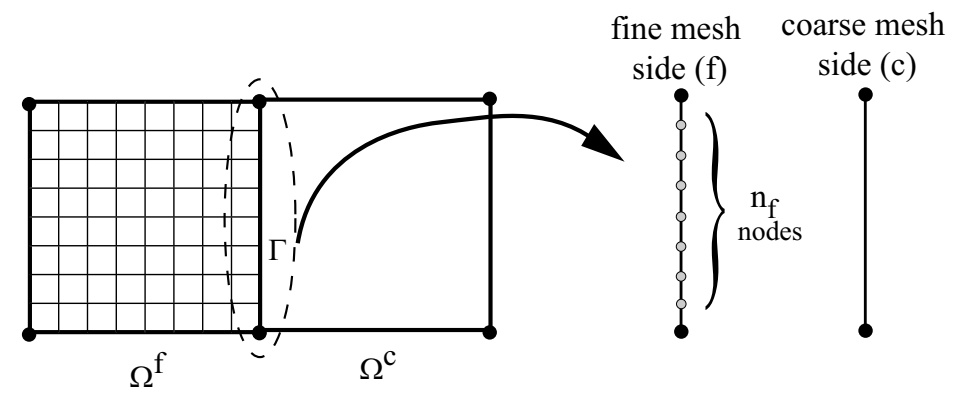

Figure 3: Interface of fine and coarse mesh

As a general rule, the mesh connection on the interface $\Gamma$, consists in satisfying the following continuity equation:

$$
\forall x \in \Gamma=\Omega^{f} \cap \Omega^{c}, \quad u_{b}^{f}(x)=u_{b}^{c}(x)
$$

where $u_{b}^{f}(x)$ and $u_{b}^{c}(x)$ are the continuous displacement fields on the fine and coarse sides of the interface $\Gamma$.

On the fine side, the continuous displacement field, $u_{b}^{f}(x)$, is defined from the underlying fine mesh and the shape functions of the corresponding elements. On the coarse side, the coarse element is built from an homogenization process, and is defined with its coarse nodes. In order to define a continuous displacement field on this coarse element, the shape functions corresponding to a classical finite element are used. In this work, since plate assemblies are considered, discrete Kirchhoff elements will be used, i.e. DKT or DKQ, see Appendix 1. With these shape functions, the displacement vectors $u_{r}^{c}$ and $u_{b}^{c}$ are denoted as:

$$
\begin{aligned}
& u_{r}^{c}=C_{c} u_{c}^{c} \\
& u_{b}^{c}=L_{r}^{c} C_{c} u_{c}^{c}
\end{aligned}
$$

where $C_{c}$ is the matrix of shape function values (interpolation matrix) on the coarse subdomain at the location of the fine mesh nodes, $u_{c}^{c}$ is the displacement vector of subdomain $\Omega^{c}$ at the coarse nodes and $L_{r}^{c}$ is the localization matrix that restrict the degrees of freedom on the global incompatible interface, the corner nodes being excluded.

Several methods were presented in the literature to connect two incompatible meshes, such as collocation [22], Mortar [23], interface element method [24] as well as other approach discussed in [25]. The weak relation for continuity of the displacement field at the subdomain interface can be written as follow:

$$
\int_{\Gamma} w(x) \cdot\left(u_{b}^{f}(x)-u_{b}^{c}(x)\right) d \Gamma=0
$$

where $w$ is a weighting function. Different choices of $w$ correspond to different types of connection.

A micro-macro interpretation of the displacement fields is presented hereafter to guide the modeling choice. For this purpose, the displacement field on the fine mesh is divided in two parts: a macroscopic part $\left(u_{b}^{f M}\right)$ and a microscopic part $\left(u_{b}^{f m}\right)$, as follow:

$$
u_{b}^{f}(x)=u_{b}^{f M}(x)+u_{b}^{f m}(x)
$$

A classical choice consists to define the macro part $\left(u_{b}^{f M}\right)$ as the interpolation of the coarse field at the nodes of the fine mesh on the interface $\left(u_{b}^{c}\right)$.

$$
u_{b}^{f M}(x)=u_{b}^{c}(x)
$$


From Equations (17),(18), it can be seen that the connection between fine and coarse meshes amounts to define locality constraints for the microscopic part of the displacement. Several approaches are discussed in [26], where macro and micro scales are superposed in the localization zone. In the work presented in this paper, micro and macro scales are separated. Moreover, the approach is embedded in the FETI-DP framework. As a consequence, due to the strong continuity at the corner nodes, the microscopic field $u_{b}^{f m}$ has to be zero on these nodes.

Two types of connection are discussed here, which are based on the displacements (kinematic) and Mortar (static) approach.

\subsection{Collocation method}

The simplest way to satisfy the equation (16) consists of using the weighting function, $w$, as Dirac $(\delta)$ functions on the all nodes of the fine mesh side of the interface. Such a procedure will be called 'collocation'.

This approach enforces the equality of the fine displacement field on one side, with the interpolation of the coarse displacement field on the other side:

$$
u_{b}^{f}\left(x_{i}\right)=u_{b}^{c}\left(x_{i}\right) \quad \text { for } \mathrm{i}=1 \text { to } n_{f}
$$

where $n_{f}$ is the number of interior interface nodes on the fine mesh side, see Figure 3.

Taking into account (17) and (18), it yields for the microscopic displacement vector:

$$
u_{b}^{f m}=0
$$

i.e. for all interior interface nodes on the fine mesh side, the micro displacement is null. Finally, from $u_{b}^{f}=B_{r}^{f} u_{r}^{f}$ and using (15), the continuity equation (19) on the interface can be written in matrix form as:

$$
A^{f} u_{r}^{f}=D^{c} u_{c}^{c}
$$

where:

$$
\begin{aligned}
A^{f} & =B_{r}^{f} \\
D^{c} & =L_{r}^{c} C_{c}
\end{aligned}
$$

and $u_{r}, u_{b}$ and $u_{c}$ are subdomain, interface and coarse nodes displacement vectors, respectively.

\subsection{Mortar method}

The Mortar method introduced in [23] is one rigorous and popular approach to enforce a weak continuity of the displacement field (16) at non-matching discrete interfaces. Within the framework of Mortar methods, the weighting function, $w$, plays the role of a Lagrange multiplier field, and a key point of the method is the space of these Lagrange multipliers which has to be chosen carefully [27, 28]. A usual choice is a modified finite element trace space of the coarsest mesh [27], with modifications concerning the end nodes of the interface [23], which are here the coarse nodes shared by the two subdomains, see e.g. [18]. In the case studied here with a coarse subdomain interface with only two nodes, if the shape functions are linear, the Lagrange multiplier has to be taken as a constant.

For plate elements used in this work, the shape functions used for membrane degrees of freedom are linear, while for bending the interpolation can be linear, quadratic, or cubic, see Appendix 1. However, for sake of simplicity, the Lagrange multiplier is chosen as a constant for each type of degree of freedom, on each interface between two subdomains. It leads us to an average displacement connecting method on the interface.

In this case, the displacement fields on the two sides of the interface satisfy the following relation:

$$
\int_{\Gamma}\left(u_{b}^{f}(x)-u_{b}^{c}(x)\right) d \Gamma=0
$$

Here the same assumption are made as in (18), i.e. the macro displacement part of the fine side equals the interpolation of the coarse displacement fields on the coarse interface side. Therefore, with considering equation (17), one obtains:

$$
\int_{\Gamma} u_{b}^{f m}(x) d \Gamma=0
$$


i.e. the average of the micro displacement part, $u_{b}^{f m}$, is null on the interface. It is recalled here that the micro displacement $u_{b}^{f m}$ is null at the corner node locations.

The discretization of the gluing condition (23) leads to the linear constraint of the degrees of freedom on two sides of the interface. The following notation is used again:

$$
\begin{aligned}
& u_{b}^{f}(x)=N(x) u_{b}^{f}=N(x) B_{r}^{f} u_{r}^{f}(x) \\
& u_{r}^{c}=C_{c} u_{c}^{c} \\
& u_{b}^{c}(x)=N(x) L_{r}^{c} u_{r}^{c}=N(x) L_{r}^{c} C_{c} u_{c}^{c}
\end{aligned}
$$

where for sake of simplicity, the continuous displacement fields, $u_{b}^{f}(x)$ and $u_{b}^{c}(x)$, are approximated using piecewise linear finite element shape function, $N(x)$, defined on the interface fine mesh side.

Note that once the coarse displacement field has been interpolated (with matrix $C_{c}$ ), the same discretizations are obtained for the coarse and fine sides of the interface. Therefore, the shape functions $N^{f}$ and $N^{c}$ on the two sides of the interface are the same and are denoted $N$.

By substituting (25) in the equation (23), one obtains the following equation:

$$
\int_{\Gamma}\left[N(x) B_{r}^{f} u_{r}^{f}-N(x) L_{r}^{c} C_{c} u_{c}^{c}\right] d \Gamma=0
$$

And in the matrix form, it yields:

$$
A^{f} u_{r}^{f}=D^{c} u_{c}^{c}
$$

with

$$
\begin{aligned}
& A^{f}=H B_{r}^{f} \\
& D^{c}=H L_{r}^{c} C_{c} \\
& H=\int_{\Gamma} N(x) d \Gamma
\end{aligned}
$$

where $H$ denotes the integral of the shape function on the interface.

Disregarding the choice of the connection method, collocation or Mortar, the relation between the degrees of freedom on the two sides of the interface can be put in similar forms (21) and (27). This relation is used for managing the incompatible interface constraints by domain decomposition method in the next Section.

\section{$5 \quad$ Numerical homogenization of a finely meshed subdomain}

In the previous section, two connection methods between incompatible displacement fields on the interface between non-matching subdomains were presented. In this section, we are concerned with the determination of an homogenized stiffness for coarse subdomains.

It is recalled there that the starting point of the method is a domain decomposition of the structure from subdomains with a fine mesh, compatible on the interface. Then, macro zones are chosen, and their corresponding subdomains are described only from their coarse nodes, see Figure 2. When a subdomain is discretized at a macroscopic (homogenized) scale, a macroscopic stiffness should be associated to it. This can be deduced from its fine discretization in a preliminary stage.

The critical step of any homogenization method lies in the localization step and concerns the way a macroscopic field loading is applied to the microscopic level. A main difficulty is the definition of suited boundary conditions to be applied to the microscopic field on the micro domain. These boundary conditions correspond to a modeling of the loadings applied by the neighboring subdomains. Therefore, to ensure consistency of the FETI-DP micro-macro approach, the previous assumptions used for connection (collocation or Mortar) are applied again. These assumptions can be explained in the framework of homogenization methods. Thus, for the collocation approach, since the micro displacement field is supposed to be zero on the subdomain boundary, this approach amounts to the strain approach of the Hill-Mandel method [29].

For the Mortar method, the micro displacement field is constrained to be zero at the corner nodes. The other boundary degrees of freedom are associated with a Lagrange multiplier which is constant. Thus there 
are similarities with the stress approach of the Hill-Mandel method [29], in which uniform stress boundary conditions are considered.

For a subdomain $s$, these boundary conditions were given by equations (15) and (19) (collocation) and equation (26) (Mortar) respectively, when fine and coarse mesh connection was treated. Since in the homogenization process only one domain with a fine mesh is considered, they can be written in the general form of the equation (21) and (27) as follows:

$$
A^{s} u_{r}^{s}=D^{s} u_{c}^{s}
$$

Moreover, the internal degrees of freedom, which are free of any loading, are condensed statically on the coarse ones. In this way, it is possible to derive a macro stiffness only defined on the coarse degrees of freedom. This homogenized stiffness is thus of a kinematic or static type, depending on the assumption used for the localization process.

For each subdomain $s$, omitting the superscript $s$ for simplification in the following, and considering the boundary conditions (29) on all the boundary of the subdomain, the equilibrium equation of this subdomain is:

$$
\forall \tilde{u}_{i}, \tilde{u}_{b}, \tilde{u}_{c}, A \tilde{u}_{b}=D \tilde{u}_{c}, \quad\left[\begin{array}{c}
\tilde{u}_{i} \\
\tilde{u}_{b} \\
\tilde{u}_{c}
\end{array}\right]^{T}\left(\left[\begin{array}{ccc}
K_{i i} & K_{i b} & K_{i c} \\
K_{b i} & K_{b b} & K_{b c} \\
K_{c i} & K_{c b} & K_{c c}
\end{array}\right]\left[\begin{array}{c}
u_{i} \\
u_{b} \\
u_{c}
\end{array}\right]-\left[\begin{array}{c}
f_{i} \\
f_{b} \\
f_{c}
\end{array}\right]\right)=0
$$

where $i, b$ and $c$ still represent the subdomain internal, interface (non-corner) and corner degrees of freedom, respectively. One can enforce the boundary conditions by Lagrange multipliers in the equilibrium equations, which leads to:

$$
\left[\begin{array}{cccc}
K_{i i} & K_{i b} & K_{i c} & 0 \\
K_{b i} & K_{b b} & K_{b c} & A^{T} \\
K_{c i} & K_{c b} & K_{c c} & -D^{T} \\
0 & A & -D & 0
\end{array}\right]\left[\begin{array}{c}
u_{i} \\
u_{b} \\
u_{c} \\
\mu
\end{array}\right]-\left[\begin{array}{c}
f_{i} \\
f_{b} \\
f_{c} \\
0
\end{array}\right]=0
$$

The macroscopic stiffness on the corner degrees of freedom of the subdomain is obtained by condensing the internal degrees of freedom $u_{i}$, then in a second stage, $u_{b}$ and $\mu$ onto the coarse degrees of freedom $u_{c}$.

As a result, one obtains the following expression for the macroscopic (homogenized) stiffness $K_{H}$ and the macroscopic (homogenized) consistent generalized force $f_{H}$ from the equilibrium equation (31):

$$
K_{H} u_{c}=f_{H}
$$

where:

$$
\begin{aligned}
& K_{H}=\left(\bar{K}_{c c}^{\star}+\bar{K}_{c b}^{\star} \bar{K}_{b b}^{\star-1} \bar{K}_{b c}^{\star}\right) \\
& f_{H}=\bar{f}_{c}^{\star}+\bar{K}_{c b}^{\star} \bar{K}_{b b}^{\star-1} \bar{f}_{b}^{\star}
\end{aligned}
$$

with:

$$
\begin{aligned}
& \bar{K}_{c c}^{\star}=K_{c c}^{\star}-K_{c b}^{\star} K_{b b}^{\star^{-1}} K_{b c}^{\star} \\
& \bar{K}_{c b}^{\star}=D^{T}+K_{c b}^{\star} K_{b b}^{\star} A^{T} \\
& \bar{K}_{b b}^{\star}=A K_{b b}^{\star^{-1}} A^{T} \\
& \bar{f}_{c}^{\star}=f_{c}^{\star}-K_{c b}^{\star} K_{b b}^{\star-1} f_{b}^{\star} \\
& \bar{f}_{b}^{\star}=A K_{b b}^{\star} f_{b}^{\star}
\end{aligned}
$$

and the terms obtained from the first condensation are:

$$
\begin{aligned}
& K_{c c}^{\star}=K_{c c}-K_{c i} K_{i i}^{-1} K_{i c} \\
& K_{c b}^{\star}=K_{c b}-K_{c i} K_{i i}^{-1} K_{i b} \\
& K_{b b}^{\star}=K_{b b}-K_{b i} K_{i i}^{-1} K_{i b} \\
& f_{c}^{\star}=f_{c}-K_{c i} K_{i i}^{-1} f_{i} \\
& f_{b}^{\star}=f_{b}-K_{b i} K_{i i}^{-1} f_{b}
\end{aligned}
$$

These notations will be used in next Section to derive the new interface problem of the FETI-DP micromacro approach. 


\section{FETI-DP MICRO-MACRO FORMULATION}

Up to this point, three types of subdomain connections on the interface can be encountered, see Figure 2:

- connection of two subdomains with compatible fine meshes,

- connection of two subdomains with compatible coarse meshes,

- connection of the subdomains with incompatible fine and coarse meshes.

The first one is classical in FETI-DP method and the second one is satisfied automatically, since the coarse nodes are common between several subdomains. For the third one, the two methods (collocation and Mortar) presented previously, can be used. The different connections on the interfaces, once assembled for the whole problem, are:

$$
\begin{array}{ll}
\sum_{s=1}^{N_{s}} B_{r}^{s} u_{r}^{s}=0 & \text { micro-micro connections } \\
\sum_{s=1}^{N_{s}}\left(A^{s} u_{r}^{s}-D^{s} u_{c}^{s}\right)=0 & \text { micro-macro connections }
\end{array}
$$

Here, the formulation of the FETI-DP micro-macro method is presented by using the connections (36), the consistent macro stiffness matrix $K_{H}$, and the generalized force vector $f_{H}$, (33).

This can be achieved by introducing a second set of Lagrange multipliers, $\mu$, for micro-macro interface, in addition to the classical Lagrange multipliers, $\lambda$, for micro-micro interface.

The unconstrained equilibrium equation for all the subdomains now reads:

$$
\begin{aligned}
\forall \tilde{u}_{r}^{s}, \tilde{u}_{c}^{s}=L_{c}^{s} \tilde{u}_{c}, \sum_{s \in N_{s f}}\left[\begin{array}{l}
\tilde{u}_{r}^{s} \\
L_{c}^{s} \tilde{u}_{c}
\end{array}\right]^{T}\left(\left[\begin{array}{ll}
K_{r r}^{s} & K_{r c}^{s} \\
K_{c r}^{s} & K_{c c}^{s}
\end{array}\right]\left[\begin{array}{c}
u_{r}^{s} \\
L_{c}^{s} u_{c}
\end{array}\right]\right. & \left.-\left[\begin{array}{c}
f_{r}^{s} \\
f_{c}^{s}
\end{array}\right]\right) \\
& +\sum_{s \in N_{s c}}\left(L_{c}^{s} \tilde{u}_{c}\right)^{T}\left(K_{H}^{s} L_{c}^{s} u_{c}-f_{H}^{s}\right)=0
\end{aligned}
$$

where $N_{s f}$ and $N_{s c}$ are the sets of subdomains with fine and coarse mesh, respectively.

It should be noted that $u_{r}^{s}$ contains all degrees of freedom of a subdomain, other than the corners degrees of freedom. So, for the macro subdomains, the degrees of freedom are restricted to $u_{c}^{s}$.

Using the interface continuity condition (36) in equation (37), it leads to:

$$
K_{r r}^{s} u_{r}^{s}+K_{r c}^{s} L_{c}^{s} u_{c}=f_{r}^{s}-B_{r}^{s^{T}} \lambda-A^{s^{T}} \mu \quad \text { for } s=1, \ldots, N_{s f}
$$

and:

$$
\begin{aligned}
\sum_{s \in N_{s f}} L_{c}^{s^{T}} K_{c r}^{s} u_{r}^{s}+\left(\sum_{s \in N_{s f}} L_{c}^{s^{T}} K_{c c}^{s} L_{c}^{s}+\sum_{s \in N_{s c}} L_{c}^{s^{T}} K_{H}^{s} L_{c}^{s}\right) u_{c}= & \\
& \left(\sum_{s \in N_{s f}} L_{c}^{s^{T}} f_{c}^{s}+\sum_{s \in N_{s c}} L_{c}^{s^{T}} f_{H}^{s}\right)+\sum_{s \in N_{s f}} L_{c}^{s^{T}} D^{s^{T}} \mu
\end{aligned}
$$

From equation (38) it follows that:

$$
u_{r}^{s}=K_{r r}^{s^{-1}}\left(f_{r}^{s}-B_{r}^{s^{T}} \lambda-A^{s^{T}} \mu-K_{r c}^{s} L_{c}^{s} u_{c}\right)
$$

Substituting equation (40) into equation (39) leads after some algebraic transformations to the new dualprimal interface problem with three unknowns (two Lagrange multipliers, $\lambda$ and $\mu$, and a primal displacement $\left.u_{c}\right)$ :

$$
\left[\begin{array}{ccc}
-K_{c c}^{\star} & F_{c l} & F_{c m} \\
F_{l c} & F_{l l} & F_{l m} \\
F_{m c} & F_{m l} & F_{m m}
\end{array}\right]\left[\begin{array}{c}
u_{c} \\
\lambda \\
\mu
\end{array}\right]=\left[\begin{array}{c}
-f_{c}^{\star} \\
d_{l} \\
d_{m}
\end{array}\right]
$$


where:

$$
\begin{aligned}
K_{c c}^{\star} & =\sum_{s \in N_{s f}} L_{c}^{s^{T}} K_{c c}^{s} L_{c}^{s}+\sum_{s \in N_{s c}} L_{c}^{s^{T}} K_{H}^{s} L_{c}^{s}-\sum_{s \in N_{s f}} L_{c}^{s^{T}} K_{c r}^{s} K_{r r}^{s^{-1}} K_{r c}^{s} L_{c}^{s} \\
F_{l l} & =\sum_{s \in N_{s f}} B_{r}^{s} K_{r r}^{s^{-1}} B_{r}^{s^{T}} \\
F_{m m} & =\sum_{s \in N_{s f}} A^{s} K_{r r}^{s^{-1}} A^{s^{T}}
\end{aligned}
$$

and

$$
\begin{aligned}
F_{c l} & =\sum_{s \in N_{s f}} L_{c}^{s^{T}} K_{c r}^{s} K_{r r}^{s^{-1}} B_{r}^{s^{T}} \\
F_{c m} & =\sum_{s \in N_{s f}} L_{c}^{s^{T}}\left(K_{c r}^{s} K_{r r}^{s^{-1}} A^{s^{T}}+D^{s^{T}}\right) \\
F_{l m} & =\sum_{s \in N_{s f}} B_{r}^{s} K_{r r}^{s^{-1}} A^{s^{T}}
\end{aligned}
$$

and finally

$$
\begin{aligned}
f_{c}^{\star} & =\sum_{s \in N_{s f}} L_{c}^{s^{T}} f_{c}^{s}+\sum_{s \in N_{s c}} L_{c}^{s^{T}} F_{H}^{s}-\sum_{s \in N_{s f}} L_{c}^{s^{T}} K_{c r}^{s} K_{r r}^{s^{-1}} f_{r}^{s} \\
d_{l} & =\sum_{s \in N_{s f}} B_{r}^{s} K_{r r}^{s^{-1}} f_{r}^{s} \\
d_{m} & =\sum_{s \in N_{s f}} A^{s} K_{r r}^{s^{-1}} f_{r}^{s}
\end{aligned}
$$

\section{SOLUTION METHOD AND NUMERICAL EXAMPLES}

Several approaches, in the context of substructure based iterative solver, already dealt with additional constraints with additional Lagrange multipliers, such as the incorporation of linear multipoint constraints $[16,17]$, and for FETI-DP formulation with Mortar constraints [30, 18].

Numerous techniques (outer iterations on $\mu$, simultaneous iterations on $\lambda$ and $\mu$ and outer iterations on $\lambda)$ presented in $[17,16,31]$, are used for solving the resulting problem (41).

In this article, the topic concerns mainly the modularity of the method. Therefore, the method of simultaneous iterations on $\lambda$ and $\mu$ is chosen, which is the direct extension of the FETI-DP method, to solve the interface problem (41) of the FETI-DP micro-macro method.

In a first step, the consistency of the FETI-DP micro-macro method is checked with patch tests. After that, in the second step, several numerical examples (cantilever beam in bending, infinite plate with a hole in traction and bending, etc.) are presented. Throughout the numerical examples, the results are compared either to an analytical solution or to a numerical reference solution (obtained with a classical finite element method, with a fine mesh in all subdomains).

Two categories of FETI-DP micro-macro methods are considered in the following, according to the assumptions used for the connection of the fine and coarse mesh and the homogenization of the coarse mesh. A static approach where the Mortar method is used, and a kinematic one if the collocation approach is chosen. It is recalled at this point that collocation corresponds to the strain approach of the Hill-Mandel homogenization theory, while the Mortar method presents similarities with the stress approach, see section 5 .

The first results of this approach were presented in [32] and [33].

\subsection{Patch tests}

Before applying the FETI-DP micro-macro method to different examples, one has to validate the two main features of the method, i.e. the connection between fine and coarse subdomains and the homogenization used to derive the coarse subdomain stiffness. To this end, patch tests are now considered, with a uniform 
state of stress and strain, and a membrane or bending loading. The examples are presented in Figure 4, with a pure traction and a pure bending case, in 2D and 3D respectively, and displacement boundary conditions in order to prevent any rigid body displacement.

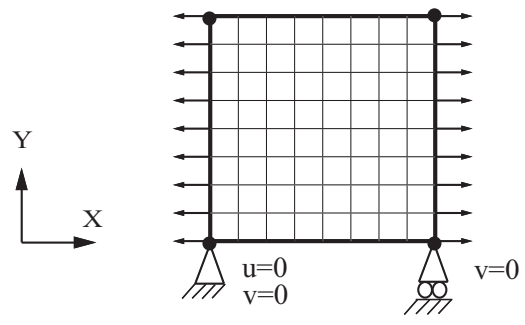

Exemple in traction

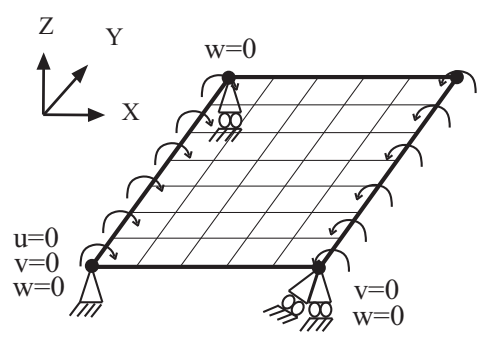

Exemple in bending

Figure 4: Patch test with one subdomain

In a first step, the problem is solved using only one coarse domain. Its macro stiffness is computed from the static or kinematic homogenization of a fine mesh, as detailed in section 5. In both cases, and for the membrane and bending patch tests, the analytical displacement solution is recovered, up to the machine precision for floating point computations.
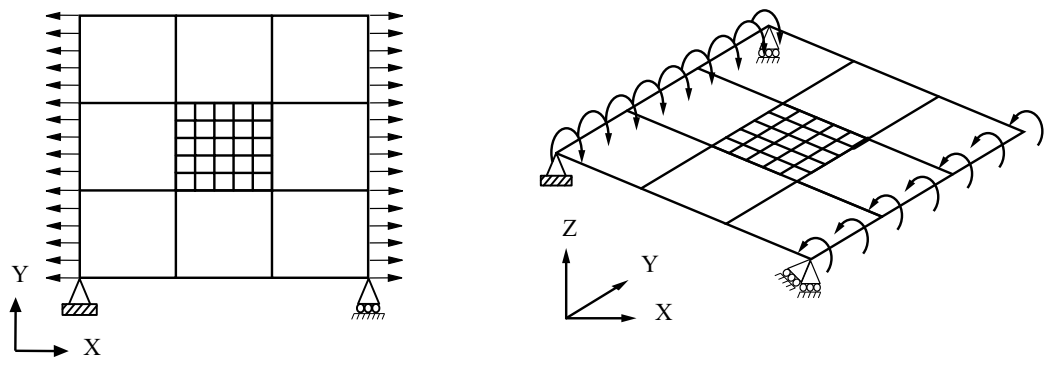

Figure 5: Patch test with an heterogeneous domain decomposition

In a second step, the same problems are considered, using the FETI-DP micro-macro proposed in this article. Thus, an heterogeneous domain decomposition is studied, with fine and coarse subdomains, as shown in Figure 5. Fine mesh is used in the subdomain located in the center, while the others are described as a coarse subdomain with the macro stiffness obtained from homogenization of a fine mesh similar to that of the central domain. Once again, the analytical displacement solution is obtained, for membrane and bending loadings, and for both types of methods, kinematic or static.

It can be noticed that the analytical solution of the problems studied in this section exhibits a uniform state of stress (generalized stress for the bending case) and a zero micro displacement on the interface, according to the definition of equations (17) and (18). These situations are fully compatible with assumptions used for the homogenization and connection between micro and macro subdomains, see equations (20) and (24). Therefore, it is logical that the the FETI-DP micro-macro approach passes the patch test.

More complicated examples will be considered in the following sections, in order to assess the approximations of the FETI-DP micro-macro method, due to the homogenization of coarse subdomains and to the connections between coarse and fine subdomains.

\subsection{End loaded cantilever beam}

In this subsection, an end-loaded cantilever beam, depicted in Figure 6, is studied. The loading consists in a parabolic shear force $P$ and a linear traction force repartition at the end of the beam as follows:

- the shear forces $(-P)$ and $(+P)$ in $y$ direction, with a parabolic distribution defined by $(43)$, are imposed at two ends of the beam (at $x=0$ and $x=L, \frac{-H}{2}<y<\frac{H}{2}$ ), 
- at $x=L$, a linear force density in the $x$ direction leads to a resultant bending moment $(-M=-P L)$ in $z$ direction, to balance the structure.

Displacement boundary conditions are also imposed at few nodes in order to prevent any rigid body motion, see Figure 6. The overall beam equilibrium provides the reactions which correspond to the force repartition shown in Figure 6. The goal of this example is to study the global response of structure by FETI-DP micro-macro method.

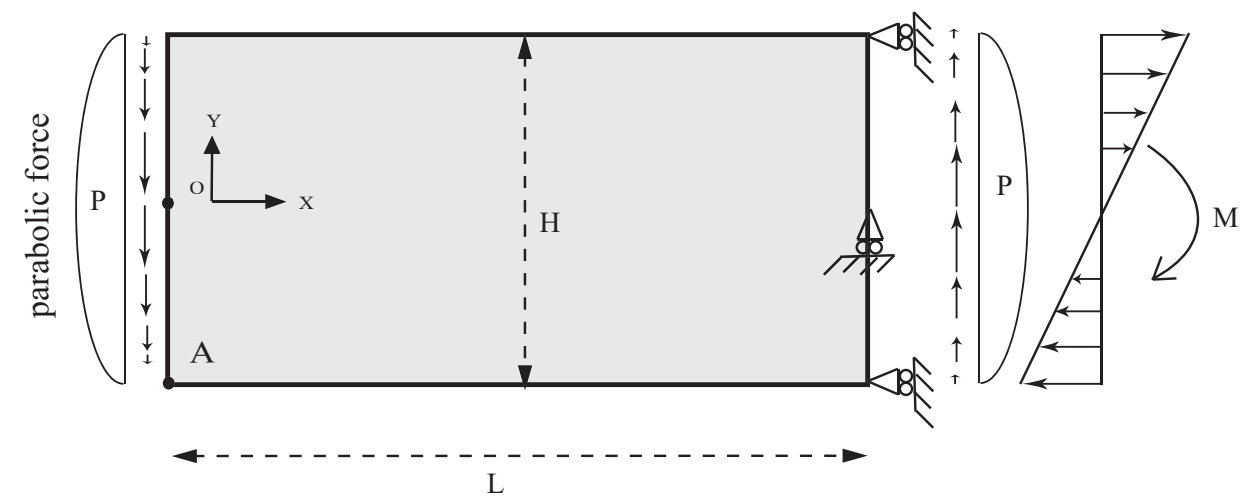

Figure 6: 2D cantilever beam in bending

This problem is bi-dimensional (plane stress) and the analytical solution is given e.g. in [34]. The displacement is:

$$
\begin{aligned}
& u(x, y)=-\frac{P y}{E I}\left[\frac{1}{2}\left(L^{2}-x^{2}\right)+\frac{(2+\nu)}{6}\left(y^{2}-\frac{H^{2}}{4}\right)\right] \\
& v(x, y)=-\frac{P}{E I}\left[\frac{L^{3}}{3}-\frac{L^{2} x}{2}+\frac{x^{3}}{6}+\frac{(4+5 \nu)}{24} H^{2}(L-x)+\frac{\nu}{2} x y^{2}\right]
\end{aligned}
$$

The stresses are given by:

$$
\begin{aligned}
\sigma_{x x}(x, y) & =\frac{P}{I} x y \\
\sigma_{y y}(x, y) & =0 \\
\sigma_{x y}(x, y) & =\frac{P}{I}\left(\frac{H^{2}}{8}-\frac{y^{2}}{2}\right)
\end{aligned}
$$

For the computations, the data used are, for the material parameters: Young's modulus $E=1 \mathrm{GPa}$ and Poisson's ratio $\nu=0.3$. For the beam geometry, a unit thickness is considered, $H=4 \mathrm{~m}$ and $L=8 \mathrm{~m}$. The loading parameters are $P=7.5 \times 10^{5} \mathrm{~N}$ and $M=6 \times 10^{6} \mathrm{~N} . \mathrm{m}$, respectively. Finally the moment of inertia is $I=H^{3} / 12$.

In this example, the stress state is not uniform on the interface and in addition the micro displacement is not zero on the micro-macro interface. These two conditions will check the homogenization and micro-macro connection in the situation more severe than the patch test.

Let us consider the deflection in vertical direction at the point $A$, see Figure 6, for the two types of mesh connection and homogenization. For different number of subdomains, one consider half of them equipped with a fine mesh, see Figure 7. The results are compared with the solution obtained with the uniform fine discretization (with a displacement $-2.78 \times 10^{-2} \mathrm{~m}$ ), see Figure 8 , while the analytic reference value is $-2.81 \times 10^{-2} \mathrm{~m}$.

It can be seen that the FETI-DP micro-macro solution is in very good agreement with the analytical solution, even with a very small number of subdomains (one coarse and one fine). In the case of 8 subdomains ( 4 coarse and 4 fine subdomains) the relative difference on the global displacement of the point $A$ is $3 \%$ for the kinematic case and $1.5 \%$ for the static case. 


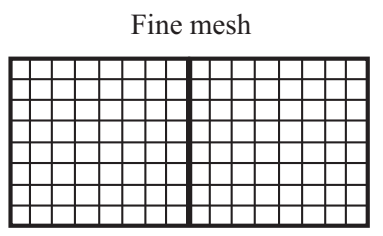

Fine and coarse mesh
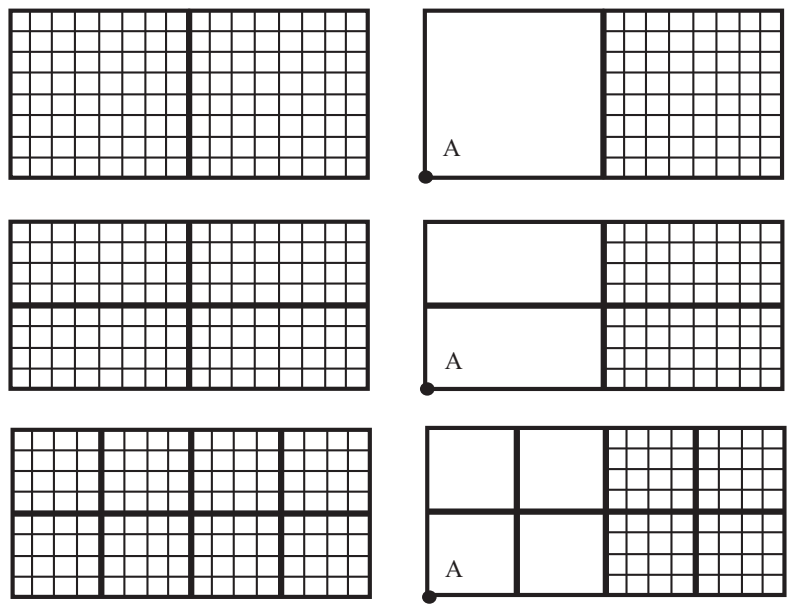

Figure 7: Decomposition in subdomains and micro-macro mesh (beam bending)

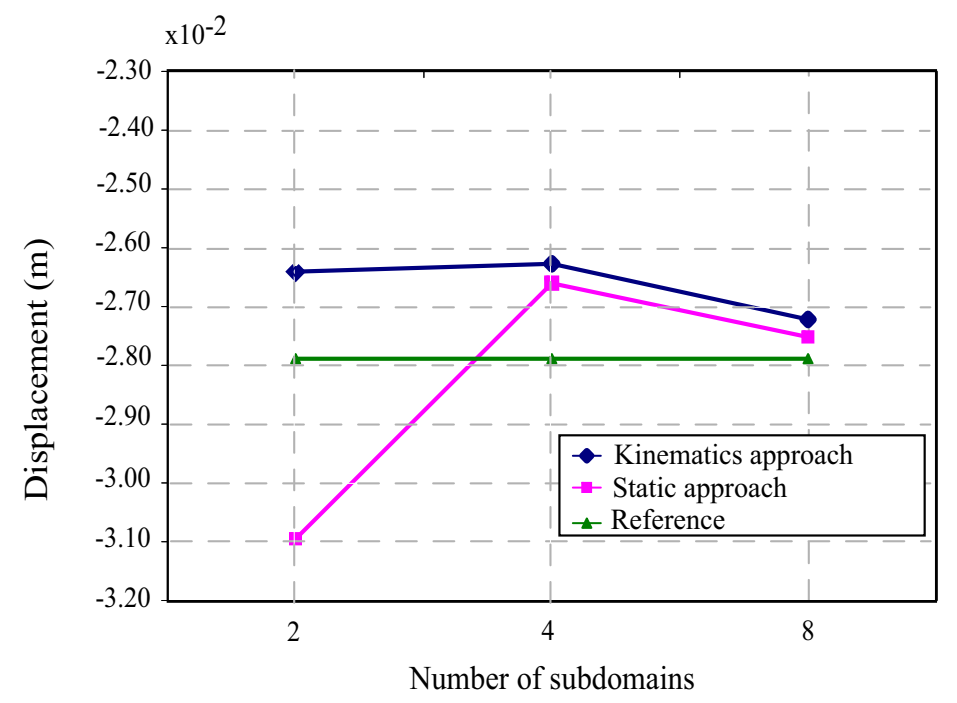

Figure 8: 2D beam with bending: displacement of the node $A$ 


\subsection{An infinite plate with a hole in traction - plane stress study}

The FETI-DP micro-macro method has been assessed in the previous example regarding overall results. The objective of the next example is to study the ability of the method to provide a good approximation of local results. To this end, the example of a plate in traction with a circular hole is considered, see Figure 9. Large stress gradients are expected around the hole, and the method will be used limiting the subdomains with a fine discretization in the vicinity of this stress concentration zone, other zones being modeled with coarse (homogenized) mesh.

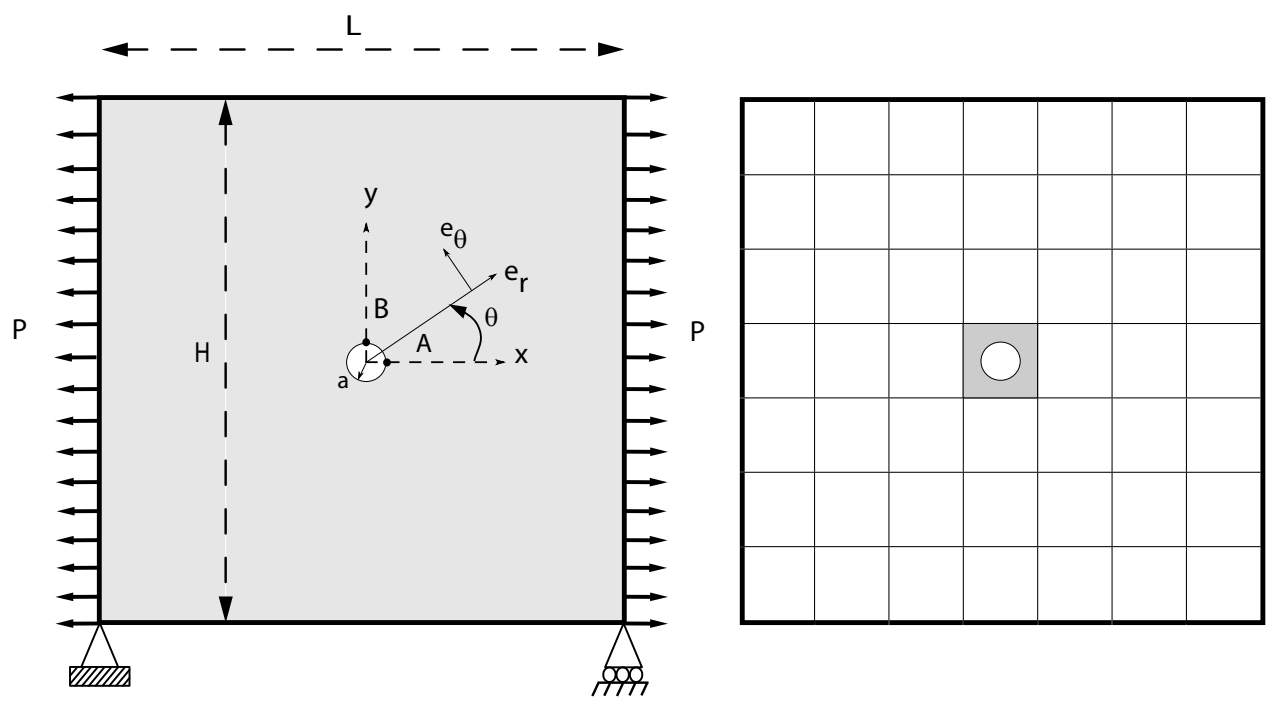

Figure 9: Plate with a hole in traction and decomposition in subdomains

The plate is subjected to a uniform tension, $P$, in the $x$ direction. The analytical solution in the case of an infinite plate is presented in [34]. The stresses are given by the following relations:

$$
\begin{aligned}
\sigma_{r r} & =\frac{P}{2}\left[\left(1-\frac{a^{2}}{r^{2}}\right)+\left(1-\frac{4 a^{2}}{r^{2}}+\frac{3 a^{4}}{r^{4}}\right) \cos (2 \theta)\right] \\
\sigma_{\theta \theta} & =\frac{P}{2}\left[\left(1+\frac{a^{2}}{r^{2}}\right)-\left(1+\frac{3 a^{4}}{r^{4}}\right) \cos (2 \theta)\right] \\
\sigma_{r \theta} & =\frac{P}{2}\left[\left(1+\frac{2 a^{2}}{r^{2}}-\frac{3 a^{4}}{r^{4}}\right) \sin (2 \theta)\right]
\end{aligned}
$$

where $r$ and $\theta$ are the polar coordinates and the angle $\theta$ is measured from the positive $x$ axis in the counterclockwise direction. The maximum values of stress occur on the hole boundary and are given by:

$$
\begin{aligned}
& \sigma_{\theta \theta}(B)=\sigma_{\theta \theta}\left(\theta=\frac{\pi}{2}\right)=3 P \\
& \sigma_{\theta \theta}(A)=\sigma_{\theta \theta}(\theta=0)=-P
\end{aligned}
$$

This analysis is based on the plane stress assumption. The isotropic material parameters are: Young's modulus $E=210 \mathrm{GPa}$, and Poisson's ration $\nu=0.3$. The following parameters are used for the plate geometry: $L=2.1 \mathrm{~m}, H=2.1 \mathrm{~m}, r=0.07 \mathrm{~m}$ and a unit thickness. The load value is $p=100 \mathrm{kN}$.

The parameters for the FETI-DP micro-macro method are $7 \times 7$ subdomains, which means that the hole diameter is close to half the subdomain length, see Figure 9. As a first step, the subdomain containing the hole is the only subdomain with a fine mesh. Since the studied plate is not infinite, a numerical reference solution is computed with a fine mesh in all the subdomains. The FETI-DP micro-macro solution is then compared to numerical and analytical reference solutions.

In order to assess the difference between the solution of the FETI-DP micro-macro method (with subscript num) and numerical reference solution (with subscript ref), we can define a relative energy norm of the error 


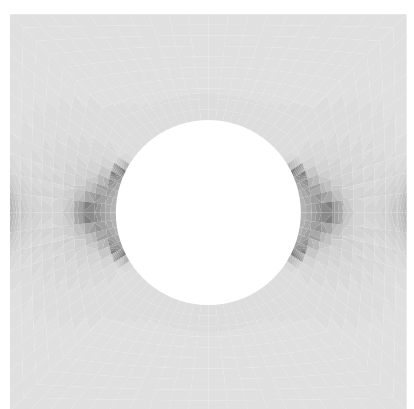

Kinematics approach

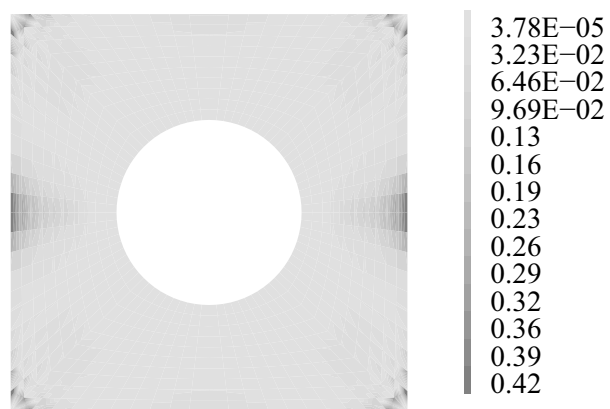

Static approach

Figure 10: Relative energy norm error, 1 micro subdomain

as:

where:

$$
r_{\Omega}=\frac{E_{\mathrm{dif}}}{E_{\mathrm{ref}}}
$$

$$
\begin{aligned}
& E_{\text {dif }}=\int_{\Omega} \varepsilon\left(u_{\text {dif }}\right): \sigma\left(u_{\text {dif }}\right) d \Omega \\
& E_{\text {ref }}=\int_{\Omega} \varepsilon\left(u_{\text {ref }}\right): \sigma\left(u_{\text {ref }}\right) d \Omega \\
& u_{\text {dif }}=u_{\text {num }}-u_{\text {ref }}
\end{aligned}
$$

This error is also computed at the finite element level, i.e. by integrating on each element, to get a local error and is displayed in Figure 10, for the central subdomain with a fine mesh. It can be seen that the static method provides more accurate results than the kinematic one. Moreover, for the static method, the error is localized on the subdomain boundary, i.e. in the vicinity of the connection zone with surrounding coarse subdomains, with a concentration at the corner nodes. However, the error decreases quickly when one moves away from the boundary. An other comparison point of view would consist in using the local contribution to the error. Since the elements of the mesh are approximatively of the same size, both plots lead to the same conclusion. These results are consistent with those obtained in [35, 36], where a static connection approach was used, without using corner continuity (note that such an additional constraint can be also prescribed in this last approach, as in [37]). On the other hand, the error obtained with the kinematic approach is lower in the vicinity of the connection zone, but increases as one gets closer to the central hole.

The deformed shape of the same subdomain is presented in Figure 11. Clearly, with the kinematic approach the interfaces are straight lines, while for static approach, the average of the displacement is null on each interface.

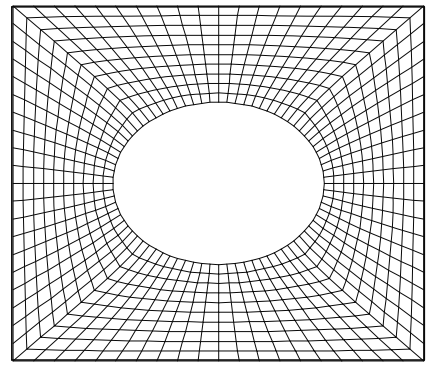

Kinematics

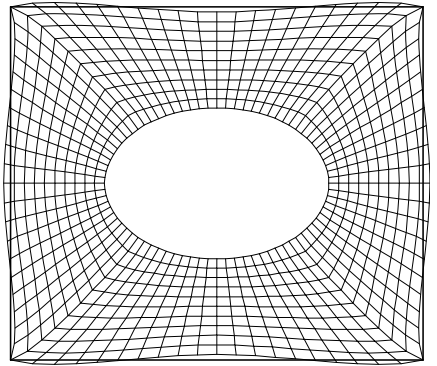

Static

Figure 11: Deformation of the central subdomain

To compare local stress values, the orthoradial stress at the points $A$ and $B$ (see Figure 9) are reported, as a function of the mesh density, see Figures 12 and 13. As mentioned previously, a significant error is 
obtained with the kinematic approach, while satisfactory results are given by the static approach, even if the size of the micro zone is only twice the hole diameter.

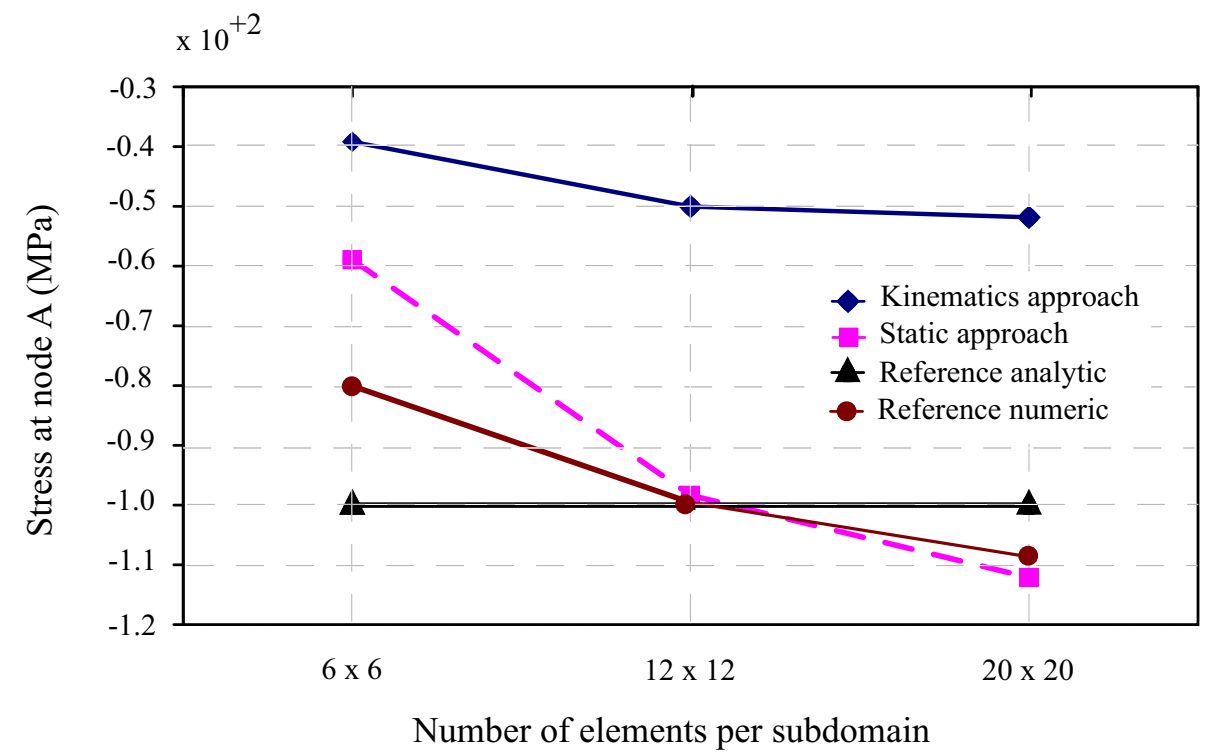

Figure 12: Infinite plate: stress at node $A\left(\sigma_{\theta \theta}\right)$

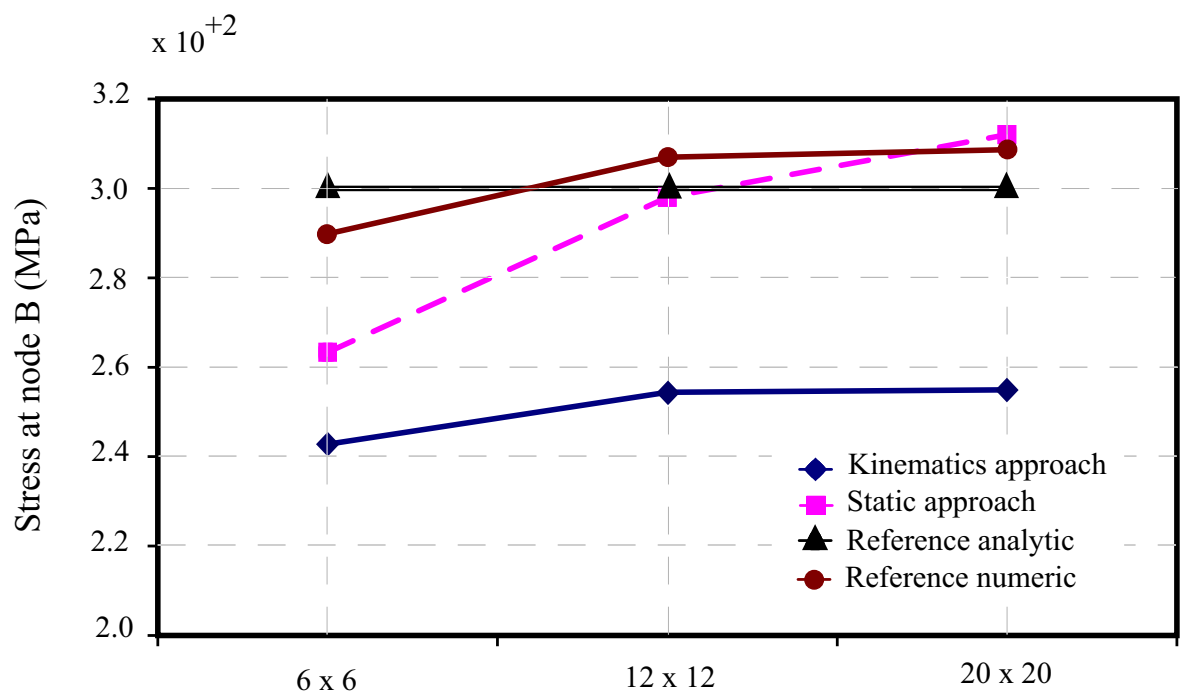

Number of elements per subdomain

Figure 13: Infinite plate: stress at node $B\left(\sigma_{\theta \theta}\right)$

It can also be checked that for both methods, the error rapidly decreases when the size of the micro zone increases. Thus, for this problem and $7 \times 7$ subdomains again, the micro zone is now extended to $3 \times 3$ subdomains at the center of the plate (instead of 1 in the previous example). The errors obtained with the FETI-DP micro-macro method are given in Figure 14, and are significantly smaller than those obtained with only one micro subdomain, see Figure 10. For the kinematic method, the error still reaches its maximum value in the vicinity of the hole boundary. 


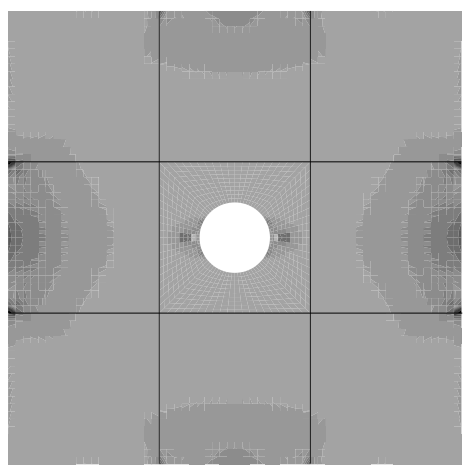

Kinematic approach

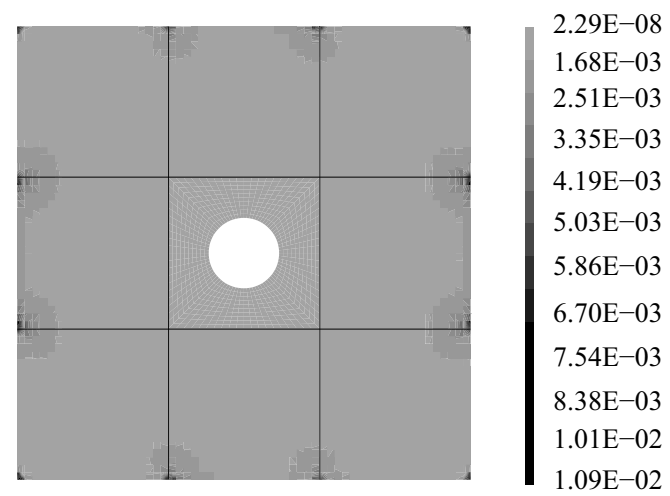

Static approach

Figure 14: Relative energy norm of error, $3 \times 3$ micro subdomains

\subsection{Plate with a hole in bending}

The same example is reused, but with a bending loading, prescribed in the same way as for the beam-like structure of Section 7.2. For the case where the micro zone is restricted to only one subdomain, the relative energy error is shown in Figure 15, which has to be compared to Figure 10 for the traction loading (membrane solution). In the bending case, the error is lower than in the traction case. The spatial distribution of the error is also different, since maximum values are obtained on the micro zone boundary. However, in the vicinity of the hole and with a closer examination of the error levels, the static approach is still slightly more accurate than the kinematic one.

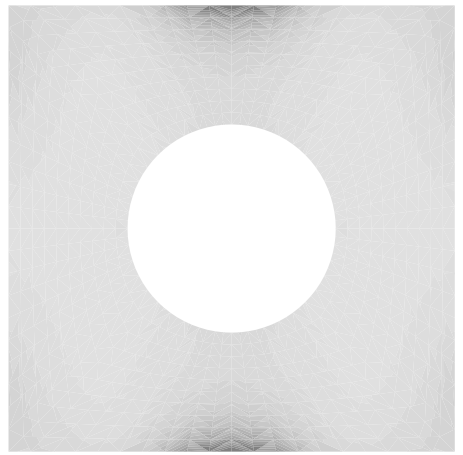

Kinematics approach

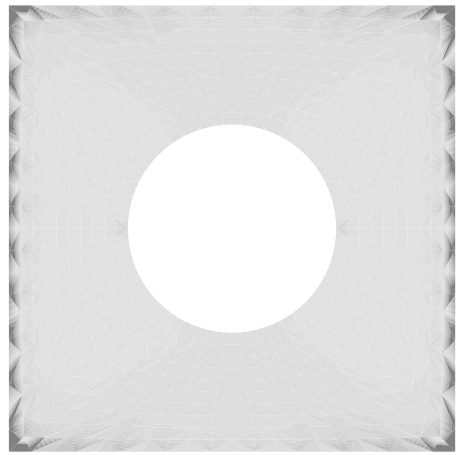

Static approach
$1.23 \mathrm{E}-03$

$1.14 \mathrm{E}-02$

$3.18 \mathrm{E}-02$

$5.22 \mathrm{E}-02$

$7.26 \mathrm{E}-02$

$9.30 \mathrm{E}-02$

0.11

0.13

Figure 15: Relative energy norm error, 1 micro subdomain, bending case

\subsection{Application to a plate assembly}

In this Section, a more complicated example, with plate assembly and structural heterogeneities (holes) is considered, see Figure 16.

The structure is subjected to an overall bending and traction loading, through a linearly varying traction force applied at one end, the other end being clamped. The material is considered as an isotropic one with the following parameters: Young's modulus $E=200 \mathrm{GPa}$ and Poisson's ration $\nu=0.3$. The following data are used for the geometry: length $L=3.0 \mathrm{~m}$, breadth $B=2.0 \mathrm{~m}$, height $H=2.0 \mathrm{~m}$, with a uniform thickness $t=0.005 \mathrm{~m}$ and a hole radius $r=0.1 \mathrm{~m}$. For this study, the structure was divided into 36 subdomains, and fine mesh is considered only in the subdomains embedding the holes, see Figure 16. Once again, a reference numerical solution is obtained with a fine mesh in every subdomain. Using this solution, the relative energy error of the FETI-DP micro-macro solution is computed, on the micro subdomains, and is given on Figure 17. 


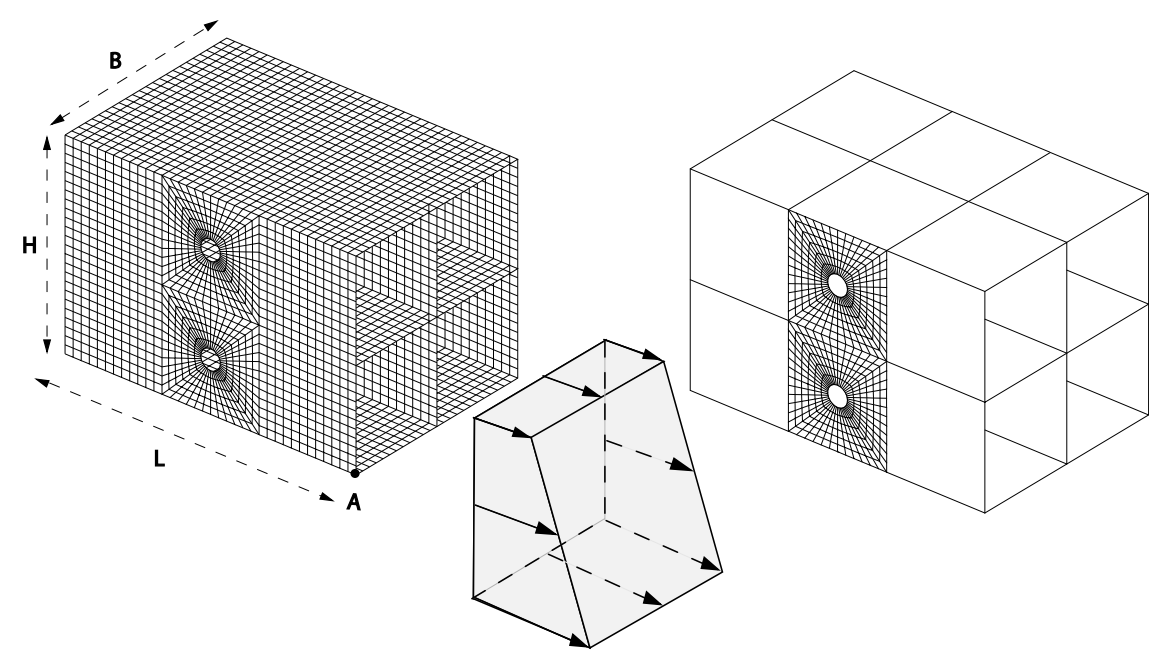

Figure 16: Plate assembly subjected to an overall bending and traction loading (left), subdomains with fine and coarse meshes (right)

As previously, the accuracy of both static and kinematic approaches is satisfactory. For the static approach, the error is mainly located close to the coarse nodes, and decreases rapidly to a small value in the vicinity of the hole. The reverse is obtained for the kinematic approach.

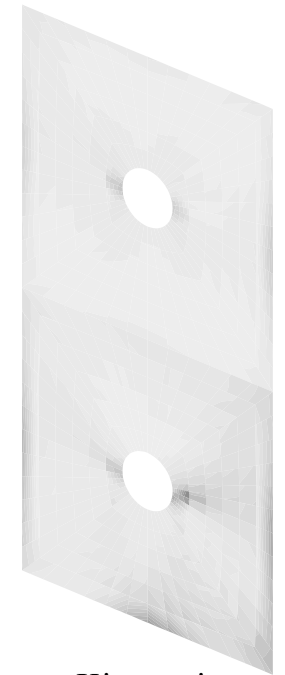

Kinematics

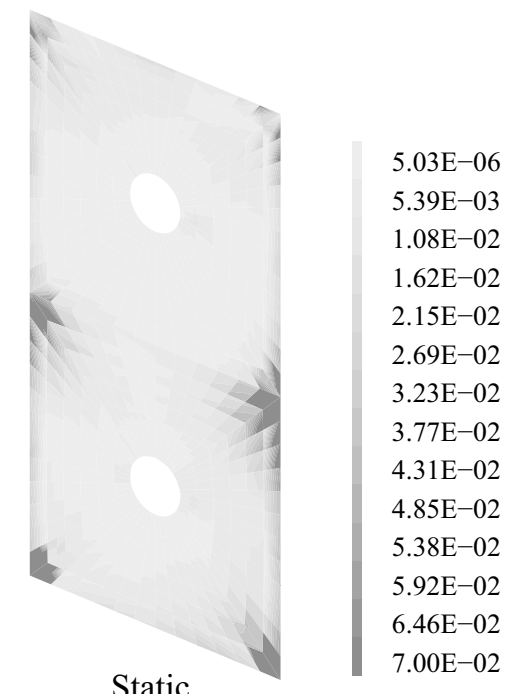

Static
$5.39 \mathrm{E}-03$
$1.08 \mathrm{E}-02$
$2.15 \mathrm{E}-02$
$2.69 \mathrm{E}-02$
$3.23 \mathrm{E}-02$
$3.77 \mathrm{E}-02$
$4.85 \mathrm{E}-02$
$5.38 \mathrm{E}-02$
$5.92 \mathrm{E}-02$
$7.00 \mathrm{E}-02$

Figure 17: Relative energy norm of error, plate assembly

\section{CONCLUSIONS}

In this article, a multi-scale extension of the FETI-DP method was presented for large-scale structural analysis. With this method, called FETI-DP micro-macro, different discretization scales can be used in the subdomains, thanks to an homogenization step. Starting from a classical domain decomposition with matching interfaces, most of the subdomains are homogenized, and the original mesh (fine mesh) is kept only in the zones where local phenomena with high stress gradients are expected. One can then optimize the computation time. 
Two methods have been proposed for the connection of micro and macro subdomains on the interface: a collocation approach and a method which can be viewed as a limit case of the Mortar method. The interface continuity is written in a weak sense, while corner continuity is enforced in a strong sense, due to the FETIDP framework. These methods contrast with other connection approaches where the continuity is written on macro interface quantities, which are not classical degrees of freedom, see e.g. [35, 36]. In the same way, two homogenization methods of the macro subdomains have been presented, with a localization process consistent with the assumptions made for the connection process. Therefore, two FETI-DP micro-macro approaches can be defined. Both were validated by using the patch tests.

The other numerical examples have shown that accurate results can be obtained from the proposed methods. For a given problem, it turns out that the results are sensitive to the size of the micro zone. In the case of a problem with structural heterogeneities, it means that the micro zone has to be large enough around them. Both static and kinematic approaches exhibit errors in the vicinity of the incompatible interfaces. However, for the static approach, this can be considered as a boundary layer effect, since the error decreases away from the interfaces. The reverse is obtained for the kinematic approach. Thus, the static approach provides more accurate results and appears to be more efficient than the kinematic one.

Concerning outlooks on this study, from a user point of view, automatic assessment of the error due to the discretization [38] and to the scale description [39] would be of particular interest, as an indication to decide to change the scale modeling. This decision could also be done as an interactive way, during iterations of the scheme to reach an adequate error level on the areas of interest. Obviously, the scale description is not limited to two levels. A third level of description could also be used, for instance for a very local analysis of fatigue or crack propagation risk, which could also be performed with coupling the plate model with a 3D model at the finest level.

Another interesting aspect to pursue this work is to adapt a specific preconditioner for the method of simultaneous iterations on $\lambda$ and $\mu$ to solve the interface problem (see section 7). Several procedures have been proposed in the literature [17], that could be tested on the problem we are interested herein.

Appendix 1

The stiffness matrix of the coarse elements are obtained from homogenization of a fine mesh. However, in order to connect coarse element with surrounding micro subdomain, a continuous displacement field has to be defined on its boundary. To this end, shape functions corresponding to a classical finite element (coarse element) are chosen.

For the membrane behavior, a linear interpolation, is a good approximation for the in-plane displacements. But for the bending case with a Discrete Kirchhoff Triangle (DKT) and/or a Discrete Kirchhoff Quadrilateral (DKQ) plate element, the interpolation functions are cubic for the out-of-plane displacement $w$, quadratic for the rotation denoted with $\theta_{n}$, and linear for the rotation denoted with $\theta_{t}$ ( $t$ is the in-plane direction of the element edge, and $n$ is the in-plane normal to the edge) [40], see Figure 18.

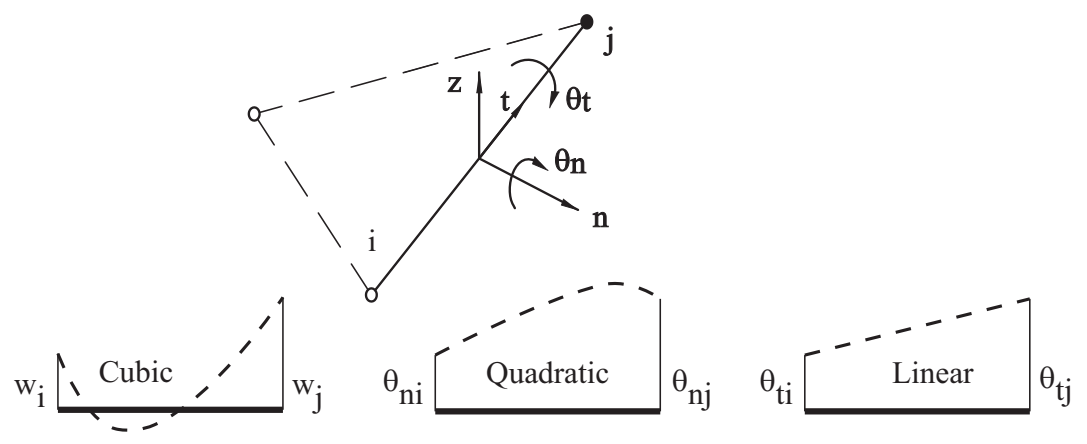

Figure 18: interpolation of the coarse field on the interface

Acknowledgements. The first author gratefully acknowledges the support of Iran's Ministry of Science, Research and Technology and SFERE exchange program in France for financial supporting during his thesis. 


\section{References}

[1] Y. Saad. Iterative Methods for Sparse Linear Systems. PWS Publishing Company, 1996.

[2] C. Farhat and F.-X. Roux. Implicit parallel processing in structural mechanics. Computational Mechanics Advances, 2:1-121, 1994.

[3] I. Parsons and J. Hall. The multigrid method in solid mechanics: Part I, algorithm description and behaviour. International Journal for Numerical Methods in Engineering, 29:719-737, 1990.

[4] I. Parsons and J. Hall. The multigrid method in solid mechanics: Part II, practical applications. International Journal for Numerical Methods in Engineering, 29:739-753, 1990.

[5] P. Vanek, M. Brezina, and J. Mandel. Convergence of algebraic multigrid based on smoothed aggregation. Numerische Mathematik, 88:559-579, 2001.

[6] P. Gosselet and C. Rey. Non-overlapping domain decomposition methods in structural mechanics. Archives of Computational Methods in Engineering, 11-4:1-59, 2005.

[7] J. Mandel. Balancing domain decomposition. Communications in Applied Numerical Methods, 9:233$241,1993$.

[8] Ch. Farhat and F.-X. Roux. A method of finite element tearing and interconnecting and its parallel solution algorithm. International Journal for Numerical Methods in Engineering, 32(6):1205-1227, 1991.

[9] L. Champaney, J.-Y. Cognard, D. Dureisseix, and P. Ladevèze. Large scale applications on parallel computers of a mixed domain decomposition method. Computational Mechanics, 19(4):253-263, 1997.

[10] K. C. Park, M. R. Justino, and C. A. Felippa. An algebraically partitioned FETI method for parallel structural analysis: Algorithm description. International Journal for Numerical Methods in Engineering, 40(15):2717-2737, 1997.

[11] D. J. Rixen, Ch. Farhat, R. Tezaur, and J. Mandel. Theoretical comparison of the FETI and algebraically partitioned FETI methods, and performance comparisons with a direct sparse solver. International Journal for Numerical Methods in Engineering, 46:501-533, 1999.

[12] Ch. Farhat, M. Lesoinne, and K. Pierson. A scalable dual-primal domain decomposition method. Numerical Linear Algebra with Applications, 7:687-714, 2000.

[13] Ch. Farhat, M. Lesoinne, P. Le Tallec, K. Pierson, and D. Rixen. FETI-DP: A dual-primal unified FETI method - Part I: A faster alternative to the two-level FETI method. International Journal for Numerical Methods in Engineering, 50(7):1523-1544, 2001.

[14] Ch. Farhat, P. S. Chen, J. Mandel, and F.-X. Roux. The two-level FETI method - Part II: Extension to shell problems, parallel implementation and performance results. Computer Methods in Applied Mechanics and Engineering, 155:153-180, 1998.

[15] M. Lesoinne. A FETI-DP corner selection algorithm for three-dimensional problems. In 14th International Conference on Domain Decomposition Methods, pages 421-428, Mexico, 2002.

[16] Ch. Farhat, C. Lacour, and D. Rixen. Incorporation of linear multipoint constraints in substructure based iterative solvers. Part 1: A numerically scalable algorithm. International Journal for Numerical Methods in Engineering, 43(6):997-1016, 1998.

[17] H. Bavestrello, Ph. Avery, and Ch. Farhat. Incorporation of linear multipoint constraints in domaindecomposition-based iterative solvers - Part II: Blending FETI-DP and mortar methods and assembling floating substructures. Computer Methods in Applied Mechanics and Engineering, 196:1347-1368, 2007.

[18] H. Kim. A FETI-DP formulation for compressible elasticity with mortar constraints. Lecture Notes in Computational Science and Engineering, 55:383-390, 2007. 
[19] P. Le Tallec. Domain decomposition methods in computational mechanics. In Computational Mechanics Advances, volume 1. North-Holland, 1994.

[20] J. H. Bramble, J. E. Pasciak, and A. H. Schatz. The construction of preconditioners for elliptic problems by substructuring, I. Mathematics of Computation, 47(175):103-134, 1986.

[21] J. Sun, P. Michaleris, A. Gupta, and P. Raghavan. A fast implementation of the FETI-DP method: FETI-DP-RBS-LNA and applications on large scale problems with localized non linearities. International Journal for Numerical Methods in Engineering, 63:833-858, 2005.

[22] D. Rixen. Substructuring and dual methods in structural analysis. Phd thesis, Université de Liège, Faculté des Sciences Appliquées, 1997.

[23] C. Bernardi, Y. Maday, and A. Patera. A new nonconforming approach to domain decomposition: The mortar element method. Collège de France Seminar, pages 13-51, 1994.

[24] H. Kim. Interface element method (IEM) for a partitioned system with non-matching interfaces. Computer Methods in Applied Mechanics and Engineering, 191:3165-3194, 2002.

[25] P.-A. Guidault and T. Belytschko. On the L2 and H1 couplings for an overlapping domain decomposition method using Lagrange multipliers. International Journal for Numerical Methods in Engineering, 70:322-350, 2007.

[26] A. Hund and E. Ramm. Locality constraints within multiscale model for non-linear material behaviour. International Journal for Numerical Methods in Engineering, 70:1613-1632, 2007.

[27] B. Herry, L. Di Valentin, and A. Combescure. An approach to the connection between subdomains with non-matching meshes for transient mechanical analysis. International Journal for Numerical Methods in Engineering, 55(8):973-1003, 2002.

[28] P. Hauret and M. Oritz. BV estimates for mortar methods in linear elasticity. Computer Methods in Applied Mechanics and Engineering, 195:4783-4793, 2006.

[29] P. Suquet. Elements of homogenization for inelastic solid mechanics. In E. Sanchez-Palencia and A. Zaoui, editors, Homogenization Techniques for Composite Media, volume 272 of Lecture Notes in Physics, pages 193-278. Springer-Verlag, Berlin, 1985.

[30] N. Dokeva. Scalable mortar methods for ellipic problems on many subregions. Phd thesis, Faculty of the graduate school University of Southern California, 2006.

[31] D. Rixen. Extended preconditioners for the FETI method applied to constrained problems. International Journal for Numerical Methods in Engineering, 54:1-26, 2002.

[32] A. Mobasher Amini, D. Dureisseix, P. Cartraud, and N. Buannic. Multi-scale domain decomposition method for structural analysis of a passenger ship. In ECCOMAS Thematic Conference: Marine 2007 - Computational Methods in Marine Engineering, Barcelona (Spain), june 2007.

[33] A. Mobasher Amini, D. Dureisseix, P. Cartraud, and N. Buannic. Analyse d'un navire à passagers avec une méthode de décomposition de domaine à plusieurs échelles. In 8e Colloque National en Calcul des Structures, Giens, mai 2007. 6 p.

[34] S. Timoshenko and J. Goodier. Theory of elasticity. Mac Graw-Hill, third edition, 1970.

[35] P.-A. Guidault. Une stratégie de calcul pour les structures fissurées : analyse locale-globale et approche multiéchelle pour la fissuration. PhD thesis, ENS Cachan, 2005.

[36] P.-A. Guidault, O. Allix, and J.-P. Navarro. A two-scale approach with homogenization for the computation of cracked structures. Computers and Structures, 85(17-18):1360-1371, 2007.

[37] P. Alart and D. Dureisseix. A scalable multiscale LATIN method adapted to nonsmooth discrete media. Computer Methods in Applied Mechanics and Engineering, 197(5):319-331, 2008. 
[38] P. Ladevèze and J.-P. Pelle. Mastering Calculations in Linear and Nonlinear Mechanics. Springer, 2004.

[39] J. T. Oden, K. Vemaganti, and N. Moës. Hierarchical modelling of heterogeneous solids. Computer Methods in Applied Mechanics and Engineering, 172:3-25, 1999.

[40] J. Batoz and G. Dhatt. Modélisation des structures par éléments finis, volume 2 - Poutres et Plaques. Hermes, Paris, 1993. 